\section{A meta-analysis of depression during pregnancy and the risk of preterm birth, low birth weight, and intrauterine growth restriction (1).}

The authors have conducted a meta-analysis to explore associations between antenatal depression and adverse birth outcomes. Published and unpublished studies, in English and non-English languages were considered if they had a prospective or longitudinal design, assessed depressive symptoms or unipolar depression by means of a screening questionnaire or structured psychiatric interview at one or more time during the pregnancy, and assessed at least one of the following adverse birth outcomes: preterm birth (PTB), low birth weight (LBW) or intrauterine growth retardation (IUGR).

862 studies were reviewed, of which 29 met inclusion criteria. Using the random-effects model, results showed that depression during pregnancy (as a categorical measure) was significantly associated with PTB $(\mathrm{RR}=1.13 ; 95 \% \mathrm{CI}, 1.06-1.21)$ and $\mathrm{LBW}$ $(\mathrm{RR}=1.18 ; 95 \% \mathrm{CI}, 1.07-1.30)$.

The authors also report that the relative risk of delivering a LBW infant was higher for women among developing countries who experienced antenatal depression, compared to their counterparts in the developed world. Women in United States from lower socio-economic background who experienced antenatal depression also had a significantly higher risk of PTB compared to their middle and upper-income women in that country.

While beyond the scope of this review, the authors suggest mechanisms by which antenatal depression may be associated with adverse birth outcomes, such as dysregulation of the hypothalamic-pituitaryadrenocortical axis in the mother stimulating release of stress hormones such as cortisol and catecholamines, which may result in placental hypoperfusion.

Significant heterogeneity across most studies, and possible publication bias were limitations of this review. The review also did not distinguish between adverse birth outcomes as a direct result of depression, versus as result of other associated factors, such as poor nutrition, poor health practices, smoking and/or alcohol use, which are often associated with depression. However the authors have attempted to control for SSRI treatment as a confounding factor.

The overall study findings indicate that identification and treatment of antenatal depression would be of benefit to both the mother and the child.
Maternal use of selective serotonin reuptake inhibitors, fetal growth, and risk of adverse birth outcomes (2).

This is a population-based study that examined associations between depressive symptoms and maternal SSRI us during pregnancy with fetal growth. 7696 mothers residing in Rotterdam were followed up during pregnancy until delivery. Maternal outcomes measures included the use of SSRIs during pregnancy and depressive symptoms during pregnancy (measured via the Brief Symptom Inventory administered at around 20 weeks of gestation). Fetal outcome measures included indicators of fetal growth during pregnancy (measured via fetal ultrasound in the first, second and third trimesters), gestational age and birth weight.

Based on these findings, the authors compared fetal outcomes in three groups of mothers: women not using SSRIs with low/no depressive symptoms $(n=7027)$, women with clinically relevant depressive symptoms not using SSRIs $(n=570)$ and women using SSRIs during pregnancy $(n=99)$. The results showed that with regards to fetal weight gain, children of mothers with depressive symptoms not using SSRIs showed a slower rate of fetal weight gain (of -4.4 g per week, 95\% CI:-6.3 to -2.4 ), whereas children of mothers using SSRI did not show a reduction of fetal growth as measured by fetal weight gain. With regards to fetal head growth - children of mothers with depressive symptoms not using SSRI showed reduced growth of head circumference (slope $\beta=-0.08 ; 95 \% \mathrm{CI}:-0.14$ to $-0.03)$; however, children of mothers using SSRIs had a more pronounced reduced head circumference growth $(\beta=-0.18 \mathrm{~mm}$ per week, $95 \% \mathrm{CI}:-0.32$ to -0.07$)$.

The authors also report that mothers using SSRIs were significantly more likely to have children born with a slightly shorter gestational duration $(\beta=-0.60$; 95\% CI:-0.97 to- 0.21 ), and to have preterm deliveries (odds ratio $=2.14 ; 95 \% \mathrm{CI}$ : 1.08 to 4.25 ), compared to controls.

The limitations of this study include the fact that depression was assessed via a screening tool for depressive symptoms, and that the severity of the depressive symptoms could not be considered in the analysis due to limitation of numbers. The reason for the reduced fetal head growth in children exposed to antenatal SSRI is also not clear - whether it is a direct effect of SSRI, or an effect of residual maternal depression, or due to depression associated epiphenomena such as smoking or alcohol use.

This study does however attempt to address the important dilemma - namely whether maternal SSRI 
treatment during pregnancy is better or worse for the fetus than untreated maternal depression. The authors highlight the fact that untreated women with depressive symptoms also have children with slower rates of fetal body and head growth. The authors recommend that clinicians carefully weigh the known risks of untreated depression during pregnancy against the possible adverse effects of SSRI, and also state that for some women with prenatal depression, the benefits of antidepressant treatment with SSRIs probably outweigh the risks.

Selected pregnancy and delivery outcomes after exposure to antidepressant medication: a systematic review and meta-analysis (3).

The aim of this study was to examine what, if any, relationship exists between prenatal antidepressant exposure and poor pregnancy or delivery outcomes. A meta-analysis was conducted of the following databases, from inception to June 2010: Medline, PsycINFO, and EMBASE. English language studies that compared pregnancy or delivery outcomes between pregnant women exposed to pharmacological antidepressants and non-exposed pregnant women were eligible for inclusion. 23 studies were eligible to be included in the final review.

Pooled data from 10 studies found that women exposed to antidepressants were at significantly increased risk of premature delivery compared to non-exposed women (pooled OR 1.55, 95\% CI 1.38 to 1.74; $\mathrm{p}<.001)$. Results also indicated that antidepressant exposure was associated with a significantly lower gestational age at birth (mean difference -0.45 weeks, $95 \% \mathrm{CI}-0.64$ to $-0.25 ; \mathrm{p}<.001$ ) and that newborns exposed to antidepressants were of significantly lower birth weight (mean difference $-74 \mathrm{~g}, 95 \% \mathrm{CI}-117$ to $-31 \mathrm{~g} ; \mathrm{p}=.001)$. There was also small but significant reductions in APGAR scores at 1 min (mean difference -.37 points) and 5 min (mean difference -.18 points) in those exposed to antidepressants.

While the findings of this study indicate that exposure to antidepressants during pregnancy is associated with a small but significant differences in fetal outcomes during pregnancy and delivery, as shown above these differences were small, and all values in the exposed groups fell within the normal range. This suggests that these effects of exposure to antidepressants may not be clinically significant. The findings were also limited by variability of quality of studies.

Perinatal episodes across the mood disorder spectrum (4).

The objectives of this study was to describe the occurrence and timing of perinatal mood episodes in women with diagnosed bipolar I disorder (BD I), bipolar II disorder (BD II) and recurrent major depression (RMD). Participants were part of 2 clinical and genetic studies of mood disorders; $26 \%$ were recruited systematically through screening community mental health teams across UK. Non-systematic recruitment was via media and patient support organizations. Women aged between 18-50 years were included in the study, and exclusion criteria included a lifetime diagnosis of intravenous drug dependency, and those who had experienced an affective illness only secondary to either alcohol or substance dependence, or a medical illness. Participants were interviewed using the Schedules for Clinical Assessments in Neuropsychiatry, and best estimate life-time diagnosis were made according to DSM-IV criteria. Information was obtained from all participants about the lifetime occurrence of pregnancy and postpartum episodes, which were rated according to DSM-IV criteria.

1212 women with bipolar disorder, and 573 women with RMD were included in the study. Women with BD-I reported approximately 1 pregnancy of 2 affected by a mood episode in pregnancy or the postpartum period (manic/mixed episode, hypomania, psychotic depression, and nonpsychotic depression); the proportion was lower in women with RMD or BDII, with both being approximately $40 \%$. When data was compared accounting for multiple pregnancies, women with BD-I had a statistically significant higher incidence of any perinatal mood episode compared with women with RMD or BD-II.

For all diagnostic categories, onset of symptoms in the postpartum period was more common than onset during pregnancy. However the onset during pregnancy was approximately twice as high for women with BD-II (18.4\%) compared to women with BD-I (8.6\%) and RMD (11.0\%). Episodes in pregnancy were equally represented across trimesters.

With regards to time of onset of the postpartum episode, women with BD I had a significantly earlier onset of a postpartum episode than did women with RMD and women with RMD reported a significantly earlier onset of a postpartum episode than did women with BD-II. $80 \%$ of perinatal episodes in women with BD I, and $72 \%$ of perinatal episodes in women with RMD had onset within the first 4 weeks of delivery, whereas only $53 \%$ of perinatal episodes in women with BD II had onset during the first 4 postpartum weeks.

A main limitation of this study is that information was collected retrospectively, and relied participant recollection of postpartum episodes.

The authors highlight that the findings indicate that BD I is associated with a higher rate of postpartum episodes, with approximately $20 \%$ of deliveries being associated with a postpartum episode of mania or psychotic depression, 25\% of deliveries being associated with an episode of nonpsychotic major depression, and overall almost $50 \%$ of deliveries being associated with a postpartum major mood disorder of some description. The authors also comment that the morbidity risk of an episode in relation to childbirth was high across all mood disorder diagnostic groups. 
ECT in Pregnancy: A Review of the Literature From 1941 to 2007 (5).

This is a review of the literature on the use of electroconvulsive therapy (ECT) during pregnancy, with the objective of discussing its risks and benefits for treating severe mental illness during pregnancy. PubMed and PsycINFO databases were searched for English or English-translated publications related to ECT administration during pregnancy.

A total of $n=339$ cases were found. The mean number of ECT treatments given was 10.8, and administration was distributed across all three trimesters. Of the cases considered, $n=68$ reported on efficacy of treatment; according to these reports, $84 \%$ of those who received ECT for depression showed at least partial response, and $61 \%$ of those who received ECT for schizophrenia or schizophreniform disorder showed at least partial remission.

The total number of fetal or neonatal abnormalities possibly related to ECT was $\mathrm{n}=11$, and the commonest fetal abnormality was transient fetal bradyarrhythmias $(2.7 \%, \mathrm{n}=8)$. One neonatal death was probably as a result of ECT, following an episode of status epilepticus in the mother secondary to ECT. There were $n=18$ reports of maternal complications following ECT, the most common adverse effect reported being uterine contractions and/or preterm labour $(3.5 \%, n=9)$. Given that the ECT current does not pass through the uterus, the authors have suggested other possible mechanisms for this side effect, including increase of oxytocin levels following ECT, or other factors such as maternal hypoxia.

The limitation of this study is the relatively small number of cases reported, possible publication bias (adverse outcomes more likely to be reported) and the lack randomized controlled trial for evaluating ECT efficacy in major depression. The review also does not examine long term effects of ECT administration.

The authors advise taking precautions to minimize the adverse effects of ECT during pregnancy, such as preoxygenation to minimize risk of fetal bradyarrhythmias, positioning of women in the late stages of pregnancy by elevating the right hip (thus minimizing aortocaval compression), and possible monitoring for uterine contractions following ECT. Overall the authors recommend ECT as a relatively safe and efficacious method of treatment for pregnant women with severe symptoms of mental illness.

\section{Declaration of interest}

None declared
Shehan Williams Senior Lecturer, Department of Psychiatry, Faculty of Medicine, University of Kelaniya, Sri Lanka Thilini Rajapakse Senior Lecturer, Department of Psychiatry, Faculty of Medicine, University of Peradeniya, Sri Lanka

\section{References}

1. Grote NK, Bridge JA, Gavin AR, Melville JL, Iyengar S, Katon WJ. A Meta-analysis of Depression During Pregnancy and the Risk of Preterm Birth, Low Birth Weight, and Intrauterine Growth Restriction. Arch Gen Psychiatry 2010;67(10):1012-1024.

2. Marroun HE, Jaddoe VWV, Hudziak JJ, Roza SJ, Steegers EAP, Hofman A, Verhulst FC, White TJH, Stricker BHC, Tiemeier H. Maternal Use of Selective Serotonin Reuptake Inhibitors, Fetal Growth, and Risk of Adverse Birth Outcomes. Arch Gen Psychiatry 2012;69(7):706-714

3. Ross LE, Grigoriadis S, Mamisashvili L, VonderPorten EH, Roerecke M, Rehm J, Dennis C, Koren G, Steiner M, Mousmanis P, Cheung A. Selected Pregnancy and Delivery Outcomes After Exposure to Antidepressant Medication. A Systematic Review and Meta-analysis. JAMA Psychiatry. 2013;70(4):436-443.

4. Florio AD, Forty L, Gordon-Smith K, Heron J, Jones L, Craddock N, Jones I. Perinatal Episodes Across the Mood Disorder Spectrum. JAMA Psychiatry. 2013;70(2):168-175.

5. Anderson EL, Reti I M. ECT in Pregnancy: A Review of the Literature From 1941 to 2007. Psychosomatic Medicine 2009;71:235-242. 